
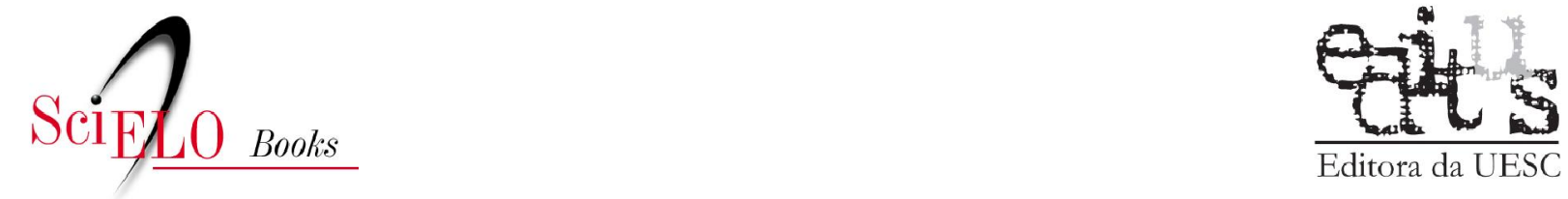

Editora da UESC

\title{
1 - As formigas Poneromorfas do Brasil
}

Introdução

\author{
Jacques H. C. Delabie \\ Rodrigo M. Feitosa \\ José Eduardo Serrão \\ Cléa S. F. Mariano \\ Jonathan D. Majer \\ (orgs.)
}

DELABIE, JHC., et al., orgs. As formigas Poneromorfas do Brasil: Introdução. In: As formigas

poneromorfas do Brasil [online]. Ilhéus, BA: Editus, 2015, pp. 9-12. ISBN 978-85-7455-441-9.

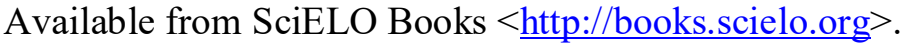

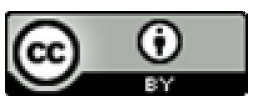

All the contents of this work, except where otherwise noted, is licensed under a Creative Commons Attribution 4.0 International license.

Todo o conteúdo deste trabalho, exceto quando houver ressalva, é publicado sob a licença Creative Commons Atribição 4.0.

Todo el contenido de esta obra, excepto donde se indique lo contrario, está bajo licencia de la licencia Creative Commons Reconocimento 4.0. 


\title{
As formigas Poneromorfas do Brasil - Introdução
}

\author{
Jacques H. C. Delabie, Rodrigo M. Feitosa, \\ José Eduardo Serrão, Cléa S. F. Mariano, Jonathan D. Majer
}

\section{Resumo}

Com 55 gêneros e 1.611 espécies atualmente descritas, o grupo de formigas "Poneromorfas" corresponde globalmente às diferentes subfamílias que, juntas, representavam as tribos da subfamília Ponerinae antes da classificação supra-genérica dos Formicidae por Bolton, publicada em 2003. Esse agrupamento artificial agrega o grande conjunto de táxons formado pelas subfamílias Amblyoponinae, Ectatomminae, Heteroponerinae, Paraponerinae, Ponerinae (no seu sentido atual) e Proceratiinae. Essas formigas possuem em comum uma grande diversidade em ambientes nativos, além de possuírem numerosas características morfológicas e comportamentais que fazem com que elas sejam muitas vezes chamadas "basais" ou "primitivas". A presente obra apresenta um conjunto de estudos originais ou revisões bibliográficas que focalizam diversas áreas do conhecimento versado às ciências biológicas, tais como taxonomia, genética, citogenética, morfologia, farmacologia, ciências do comportamento, agronomia, ecologia, biogeografia e biomonitoramento. Esses estudos foram realizados no decorrer do projeto "Rede Multidisciplinar de Estudos sobre Formigas Poneromorfas do Brasil", que recebeu apoio das agências de financiamento à pesquisa Fundação de Amparo à Pesquisa do Estado da Bahia (FAPESB) e Conselho Nacional de Desenvolvimento Científico e Tecnológico (CNPq).

DELABIE, Jacques H. C.; FEITOSA, Rodrigo; SERRÃO, José Eduardo; MARIANO, Cléa; MAJER, Jonathan. As formigas Poneromorfas do Brasil - Introdução. In: DELABIE, Jacques H. C. et al. As formigas poneromorfas do Brasil. Ilhéus: Editus, 2015. p. 9-12. 
The poneromorph ants of Brazil: Introduction - With 55 genera and 1,611 species currently described, the ants collectively referred to as "Poneromorphs" globally correspond to those subfamilies that formerly represented tribes of the subfamily Ponerinae, prior to the supra-generic classification of Formicidae by Bolton, published in 2003. This artificial grouping aggregates a large set of taxa that are now contained within the subfamilies Amblyoponinae, Ectatomminae, Heteroponerinae, Paraponerinae, Ponerinae (in its modern sense) and Proceratiinae. These ants share a great diversity in native environments and possess many morphological and behavioural characteristics that lead to them being considered "basal" or "primitive". This book presents a set of original studies and literature reviews that focus on different areas of knowledge, such as taxonomy, genetics, cytogenetics, morphology, pharmacology, behavioural sciences, agronomy, ecology, biogeography and biomonitoring. These studies were carried out as part of the project entitled "Multidisciplinary Study Network on Poneromorph Ants of Brazil", which received support from the research funding agencies Fundação de Amparo à Pesquisa do Estado da Bahia (FAPESB) and Conselho Nacional de Desenvolvimento Científico e Tecnológico (CNPq).
Durante os quarenta últimos anos, inúmeras pesquisas sobre a biologia das formigas ( $\mathrm{Hy}-$ menoptera: Formicidae) permitiram enormes progressos na compreensão do extraordinário sucesso ecológico e evolutivo desses insetos sociais (WARD, 2014). Uma das consequências lógicas foi uma verdadeira revolução na taxonomia e filogenia desta família. Graças à contribuição crescente de novas tecnologias para aquisição de fontes originais de dados biológicos (p.e. citogenética, biologia molecular e microscopia eletrônica), assim como através do uso generalizado do método cladístico nos estudos de sistemática, quase todas as subfamílias de formigas sofreram revisões recentes e, por consequência, novas classificações foram propostas. Nesse contexto, Bolton (2003) publicou uma nova classificação supra-genérica dos Formicidae. As subfamílias de formigas foram agrupadas em grupos sistemáticos informais, cujo prefixo é o da mais antiga subfamília descrita em cada grupo (p. e., formicomorfos, myrmicomorfos, poneromorfos, entre outros.). O que designamos "poneromorfas" a seguir constitui um desses grupos, objeto focal da obra aqui apresentada.

O grupo "poneromorfo" foi criado a fim de designar o grande conjunto de táxons formado pelas subfamílias Amblyoponinae, Ectatomminae, Heteroponerinae, Paraponerinae, Ponerinae [sensu stricto, Bolton (2003)], e Proceratiinae. Taxonomicamente, com 55 gêneros e 1.611 espécies descritas
(BOLTON, 2014), o grupo "poneromorfo" corresponde globalmente às diferentes tribos que, juntas, estabeleciam a subfamília Ponerinae na sua antiga definição [sensu lato, Bolton (1990a,c)]. Morfologicamente, todas as suas espécies se caracterizam principalmente pela fusão tergo-esternal do quarto segmento abdominal (BOLTON, 1990a, 2003; WARD, 1994). Estas formigas se distribuem em todas as regiões zoogeográficas do mundo e ocupam uma grande diversidade de nichos ecológicos, sendo tanto pequenas e crípticas como grandes e notáveis (OUELETTE et al., 2006). Evolutivamente, apesar das subfamílias poneromorfas formarem um agrupamento muito heterogêneo de táxons, tanto morfologicamente como do ponto de vista comportamental e social (WHEELER, 1910; HÖLLDOBLER; WILSON, 1990; WILSON; HÖLLDOBLER, 2005; OUELETTE et al., 2006), estas são, sobretudo, consideradas como globalmente "primitivas" dentro das formigas. Por isso, vários estudos filogenéticos utilizando dados morfológicos (GRIMALDI et al., 1997; HASHIMOTO, 1996a, 1996b; KELLER, 2000, 2011; WARD, 1994) e/ou moleculares (SAUX et al., 2004; SULLENDER; JOHNSON, 1998; WARD; BRADY, 2003; WARD; DOWNIE, 2005; BRADY et al., 2006; OUELETTE et al., 2006; SCHMIDT; SHATTUCK, 2014) apontam que as poneromorfas são de fato um agrupamento artificial.

Apesar da parafilia, as poneromorfas compartilham os seguintes caracteres morfológicos 
por convergência: (1) orifício da glândula metapleural nunca coberto por uma franja dorsal da cutícula; (2) lobos propodeais presentes; (3) cintura formada por um segmento (pecíolo), separado posteriormente do segmento abdominal III por uma constrição; (4) esternito do hélcio retraído e coberto pelo tergito; (5) segmento abdominal IV com pré-escleritos e usualmente uma constrição em forma de anel presente; (6) espiráculos nos segmentos abdominais V a VII cobertos pelas margens posteriores dos tergitos anteriores; (7) aparelho de ferrão presente e frequentemente bem desenvolvido. Além desses caracteres morfológicos, há outros de natureza comportamental ou ecológica que, também por convergência, sugerem certa unidade funcional entre estas formigas, justificando o esforço particular em estudá-las.

Atualmente, Formicidae é composta por 16 subfamílias, englobando cerca de 13.000 espécies descritas, distribuídas em aproximadamente 330 gêneros (BOLTON, 2014). Entre os invertebrados, as formigas formam, em geral, um dos grupos melhor estudados pelos entomologistas. Entre as formigas, as poneromorfas certamente possuem as espécies que mais chamam a atenção dos pesquisadores, em razão da sua diversidade em ambientes nativos das regiões tropicais, mas também, principalmente, em razão das características morfológicas e comportamentais menos derivadas ("mais primitivas") que elas possuem em comum, fazendo com que esses animais estejam entre os organismos sociais mais estimulantes intelectualmente a estudar. Foi observado que, no Brasil, há uma abundante literatura científica regularmente produzida sobre essas formigas, fruto das numerosas pesquisas realizadas. No entanto, a maioria dessas é feita individualmente ou independentemente por pesquisadores ou grupos isolados cientificamente ou mesmo geograficamente. Nossa intenção foi organizar os esforços desses grupos com interesses convergentes numa rede onde foram compartilhados o conhecimento e a experiência dos colaboradores, agregando interesses de entomólogos, agrônomos, etólogos, ecólogos, microbiologistas, biólogos forenses, geneticistas, biólogos moleculares, citogeneticistas entre outros; a maioria com experiência já comprovada sobre aspectos da biologia das formigas poneromorfas. Em consequência, as pesquisas desenvolvidas interessam as mais diversas áreas das ciências biológicas, tais como genética, microbiologia, medicina, agronomia, ecologia, biogeografia, ciências do comportamento e farmacologia.
As formigas "poneromorfas" geralmente vivem em pequenas populações, com pouca variação morfológica entre operárias, praticamente com ausência de polimorfismo, castas de fêmeas (=rainha e operária) pouco diferenciadas e capacidade de recrutamento limitada, características consideradas basais na história evolutiva do grupo. Outro ponto de interesse é que as espécies pertencentes ao grupo são formigas consideradas predadoras por excelência (JIMÉNEZ et al., 2008), fato que tem numerosas implicações práticas; por exemplo, o conhecimento sobre o seu potencial como predadoras de insetos e outros organismos nocivos às lavouras, pode levar a importantes avanços em estudos sobre a redução de prejuízos agrícolas provocados por pragas em diversos cultivos. Além disso, as formigas pertencentes a esse grupo, em geral, são bem mais frequentes em áreas conservadas do que em áreas antropizadas. Desse modo, estudos relacionados a esse grupo podem ajudar no monitoramento da qualidade ambiental em áreas avaliadas e fornecer argumentos para embasar medidas legais visando à implantação de unidades de conservação, por exemplo.

\section{Referências}

BOLTON, B. Abdominal characters and status of the Cerapachyinae ants (Hymenoptera, Formicidae). Journal of Natural History, v. 24, 53-68, 1990a.

BOLTON, B. Army ants reassessed: the phylogeny and classification of the doryline section (Hymenoptera,

Formicidae). Journal of Natural History, v. 24, 1339$1364,1990 \mathrm{~b}$.

BOLTON, B. Synopsis and classification of Formicidae. Florida. Memoirs of the American Entomological

Institute, Gainesville. 2003.370 p.

BOLTON, B. An online catalog of the ants of the world. Available from $<$ http://antcat.org $>$. (accessed [17.x1.2014]).

BRADY, S. G.; SCHULTZ, T. R.; FISHER, B. L.; Ward, P. S. Evaluating alternative hypotheses for the early evolution and diversification of ants. Proceedings of the National Academy of Sciences of the United States of America, 13(48): 18172-18177, 2006.

GRIMALDI, D.; AGOSTI, D.; CARPENTER, J. M. New and rediscovered primitive ants (Hymenoptera: Formicidae) in Cretaceous amber from New Jersey, and their phylogenetic relationships. American Museum Novitates, 3208: 1-43, 1997. 
HASHIMOTO, Y. Skeletomuscular modifications associated with the formation of an additional petiole on the anterior abdominal segments in aculeate Hymenoptera. Japanese Journal of Entomology, 64:340-356, 1996.

HÖLLDOBLER B., WILSON, E. O. The Ants. Harvard University Press USA. 1990.732p.

JIMÉNEZ, E.; FERNÁNDEZ F.; Milena Arias T.; LOZANO-ZAMBRANO F. H. (Eds.). Sistemática, Biogeografía y Conservación de las Hormigas Cazadoras de Colombia, Instituto de Investigación de Recursos Biológicos Alexander von Humboldt, Bogotá, Colombia, 241-283. ISBN 978-958-834318-1

KELLER, R. A. Cladistics of the tribe Ectatommini (Hymenoptera: Formicidae): a reappraisal. Insect Systematics; Evolution, 31 (1), 59-69, 2000.

KELLER, R. A. A phylogenetic analysis of ant morphology (Hymenoptera: Formicidae) with special reference to the poneromorph subfamilies. Bulletin of the American Museum of Natural History, 355: 1-90, 2011.

OUELLETTE, G. D.; FISHER B. L.; GIRMAN D. J. Molecular systematics of basal subfamilies of ants using 28S rRNA (Hymenoptera: Formicidae). Molecular Phylogenetics and Evolution, 40: 359-369, 2006.

SAUX, C.; FISHER, B. L.; SPICER, G. S. Dracula ant phylogeny as inferred by nuclear $28 \mathrm{~S}$ rDNA sequences and implications for ant systematics (Hymenoptera: Formicidae: Amblyoponinae). Molecular Phylogenetics and Evolution, 33: 457-468, 2004.
SCHMIDT, C. A.; S. O. SHATTUCK. The higher classification of the ant subfamily Ponerinae (Hymenoptera: Formicidae), with a review of Ponerine ecology and behavior. Zootaxa, v. 3817, n. 1, p. 001 $242,2014$.

SULLENDER, B.W., JOHNSON, M. J. A preliminary molecular phylogeny for the Formicidae. Social insects at the turn of the millenium. In: Schwarz, M.P., Hogendoorn K. (Eds.), Proceedings of the XIII International Congress of IUSSI, Adelaide Australia. 1999. p. 535.

WARD, P. S. Adetomyrma, an enigmatic new ant genus from Madagascar (Hymenoptera: Formicidae), and its implications for ant phylogeny. Systematic Entomology, 19: 159-175, 1994.

WARD, P. S. The phylogeny and evolution of ants. Annual Review of Ecology, Evolution, and Systematics, 45: 23-43, 2014.

WARD, P. S.; BRADY, S. G. Phylogeny and biogeography of the ant subfamily Myrmeciinae (Hymenoptera: Formicidae). Invertebrate Systematics, 17: 361-386, 2003.

WARD P. S.; DOWNIE, D. A. The ant subfamily Pseudomyrmecinae (Hymenoptera: Formicidae): phylogeny and evolution of big-eyed arboreal ants. Systematic Entomology, 30: 310-335, 2005.

WHEELER, W. M. Ants: Their structure, development and behavior. New York, Columbia University Press. 1910.

WILSON, E. O.; HÖLLDOBLER, B. Eusociality: origin and consequences. Proceedings of the National Academy of Sciences of America, 102 (38): 1336713371, 2005. 